\title{
A Concept of the Interactive Information and Modeling System for Gas Dynamics and Multidisciplinary Research
}

\author{
P. Silvestrov ${ }^{1}$, O. Bessonov ${ }^{2}$, A. Rybakov ${ }^{1}$ \\ ${ }^{1}$ Tactical Missiles Corporation \\ Korolev, Moscow district, 141080, Russia \\ ${ }^{2}$ Institute for Problems in Mechanics of the Russian Academy of Sciences \\ Moscow, 119526, Russia \\ pavelsilvestrov@yandex.ru
}

\begin{abstract}
This paper describes the general idea and the implementation of the Interactive information and modeling system - an integrated environment that combines computational modules for modeling the aerodynamics and aerothermodynamics of re-entry space vehicles with the large collection of different information materials on this topic. This system provides interactive mechanisms for performing numerical simulation of processes around a vehicle and analysis of the results. The paper describes and illustrates the internal organization and the composition of the system. Examples of the computational and information output are presented together with the simplified working scenario. The system has the unified implementation for Windows and Linux operation systems and can be deployed on any modern high-performance personal computer.
\end{abstract}

Keywords: gas dynamics, computational aerodynamics, interactive information and simulation system, computer graphics.

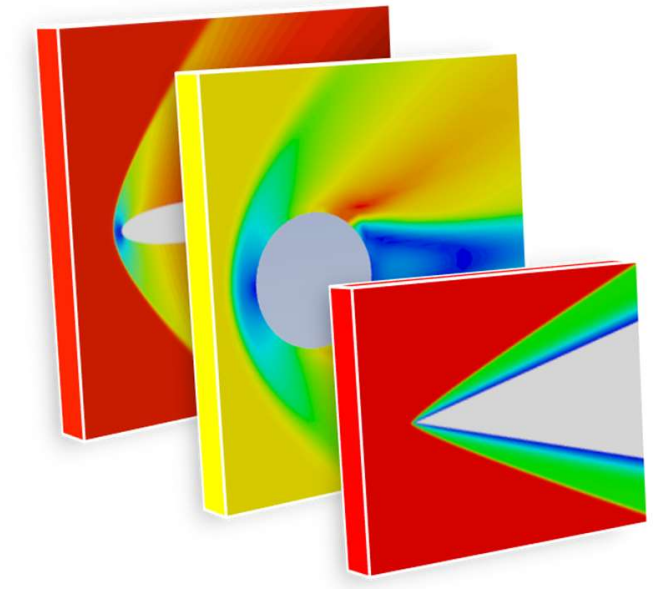

Examples of the of Mach number distribution on the surfaces of primitive objects

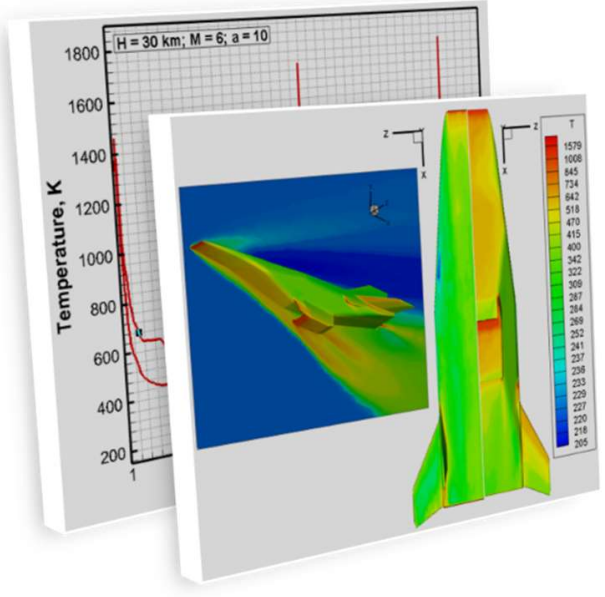

Example of the temperature distribution on the surface of a vehicle 


\title{
О концепции интерактивной информационно- расчетной системы для задач газовой динамики и междисциплинарных исследований
}

\author{
П.В.Сильвестров ${ }^{1}$, О.А.Бессонов ${ }^{2}$, А.Н.Рыбаков ${ }^{1}$ \\ ${ }^{1}$ Корпорация «Тактическое ракетное вооружение», \\ Россия, Московская обл., Королев, 141080, ул. Ильича, д. 7 \\ ${ }^{2}$ Институт проблем механики им. А.Ю. Иилинского Российской академии наук, \\ Россия, Москва, 119526, проспект Вернадского, д.101, корп. 1 \\ pavelsilvestrov@yandex.ru
}

\begin{abstract}
Аннотация
В статье описываются концепция и основные подходы к реализации Интерактивной информационно-расчетной системы - интегрированной среды, позволяющей объединять вычислительные модули для моделирования аэродинамики и аэротермодинамики спускаемых космических аппаратов с большим количеством информационных материалов по данной тематике. Система реализует интерактивный механизм проведения расчетов и анализа результатов. В работе описаны и проиллюстрированы внутренняя иерархия и интерфейс системы. Приведены примеры вычислительного и информационного режимов работы с системой. Система имеет кроссплатформенную реализацию для операционных систем Windows и Linux, что позволяет ее использовать на различных современных высокопроизводительных персональных компьютерах.
\end{abstract}

Ключевые слова: газовая динамика, вычислительная аэродинамика, интерактивная информационно-расчетная система, компьютерная графика.

\section{1. Введение}

Эффективное развитие высокотехнологических отраслей промышленности существенно зависит от информационно-компьютерных технологий. Такие технологии широко используются в странах, разрабатывающих летательные аппараты (США, Франция, Япония и т.д.), для предсказания характеристик и оптимизации параметров летательных аппаратов на этапе проектирования, анализа данных, в ходе наземной и летной отработки, а также прогностических исследований по данной тематике. Однако точность вычислений зачастую не соответствует современным требованиям.

Последние достижения в области суперкомпьютерных технологий и математического моделирования сложных динамических процессов для спускаемых космических аппаратов [1-2] обеспечивают возможность создания компьютерных кодов, использование которых позволяет значительно сократить количество наземных и летных испытаний, а также снизить влияние сопутствующих рисков и повысить экономическую эффективность работ в процессе разработки оптимального интегрального облика инженерных решений.

В настоящее время не существует единого программного пакета для полноценного междисциплинарного моделирования взаимодействия космического аппарата с обтекающим газом. При этом ведущие научно-исследовательские институты и конструкторские бюро в области аэрокосмических исследований имеют богатый опыт решения отдельных частей 
этой глобальной проблемы с использованием компьютерных кодов собственной разработки [3-11].

Таким образом, возникает задача систематизации и структурирования как существующих, так и новых компьютерных кодов в единый программный комплекс с модульной архитектурой.Такой комплекс позволит решить задачу моделирования различных видов взаимодействия (механического, термического, радиационного и химического) летательного аппарата с окружающей средой в течение всего полета.

Интеграционный комплекс должен включать в себя набор программ для расчета аэродинамики (аэротермодинамики) спускаемых космических аппаратов и элементов их конструкции, а также инструменты и средства, обеспечивающие:

- $\quad$ выбор прототипа модели из банка прототипов;

- $\quad$ ввод и подготовку параметров расчета;

- подготовку, выбор и визуализацию поверхностных и пространственных сеток;

- получение необходимых физико-химических характеристик веществ;

- $\quad$ выполнение компьютерных вычислений и контроль за их выполнением;

- графический и числовой представления результатов расчетов;

- $\quad$ возможность изучения информационных ресурсов по теме моделирования.

В рамках создания ИИРС разрабатываются технологии, соответствующие передовому мировому уровню. Новый программный комплекс позволит:

- $\quad$ определять аэродинамические и тепловые нагрузки на компоновку спускаемых космических аппаратов и расчет внешних течений;

- $\quad$ рассчитывать термогазодинамические характеристики внутренних течений с учетом процессов горения;

- $\quad$ моделировать высокотемпературные потоки в ударном слое около летательного аппарата синтегрированной силовой установкой;

- $\quad$ моделировать взаимодействие высокотемпературных газов с теплозащитными покрытиями аппарата;

- $\quad$ моделировать нестационарные течения в интегрированной силовой установке, в том числе процессы воспламенения в камере сгорания;

- $\quad$ моделировать неравновесные химические и фотохимические процессы и формирование кластеров и аэрозольных частиц в выхлопных струях силовой установки;

- $\quad$ моделировать сигнатуры летательного аппарата и способы подавления естественных и искусственных атмосферных возмущений.

Полнофункциональная система физико-математических моделей аэротермодинамики позволит проводить численное моделирование внешнего обтекания спускаемых космических аппаратов и их ключевых элементов (кромки корпуса и крыльев, носового обтекателя, силовой установки). В частности, моделирование ключевых элементов аппарата позволяет добиться более высокой точности расчета, что повышает надежность моделирования аппарата в целом.

Программный комплекс также обеспечивает доступ к банку стандартных тестовых задач, сформированных на основе наземных и летных экспериментальных данных. Тестовые задачи используются для верификации и валидации компьютерных кодов, входящих в состав системы.

Кроме того, в состав комплекса входит база данных по газовой динамике и междисциплинарным исследованиям. Эта база данных содержит информацию о физико-химических свойствах газов на высоких скоростях, кинетике сгорания компонентов топлива, тепловых, термодинамических и спектрально-оптических характеристиках веществ, а также каталитических свойствах поверхностей.

В данной статье приведены ключевые технологии создания интерактивной информационно-расчетной системы (ИИРС) [12]. Информационно-справочные данные, используемые в 
системе, получены в ходе предыдущих исследований в области радиационной газовой динамики, высокоскоростной аэротермодинамики и физико-химической механики неравновесных сред и высокотемпературной термодинамики (см., например, [6-11]).

\section{2. Состав информационно-расчетной системы}

Интерактивная информационно-расчетная система, рассматриваемая в данной статье, представляет собой интегрированную диалоговую систему, развернутую на высокопроизводительном компьютере и содержащую набор прикладных расчетных программ и средств взаимодействия между ними, объединенных единой графической оболочкой.

Информационно-расчетное наполнениеИИРС включает в себя:

- $\quad$ набор программ для расчета аэродинамики (аэротермодинамики) прототипов и элементов конструкции;

- $\quad$ средства для подготовки расчета (выбор прототипа из банка данных, ввод и подготовка параметров расчета, выбор и визуализация поверхностных и пространственных сеток);

- средства для вывода результатов (графического и числового представления результатов, текстовой и презентационной информации, верификации и валидации результатов);

- $\quad$ информационный банк, содержащий статьи, отчеты, подборки, графики, видеофрагменты и прочие информационные материалы по данной тематике, необходимые для сопровождения проводимых расчетов и для анализа получаемых результатов;

- $\quad$ банк данных характеристик веществ с возможностью выборки и подготовки информации, необходимой для проведения расчетов.

Программный комплекс может быть развернут на высокопроизводительном компьютере, работающем под управлением операционной системы Windows или Linux.

Единая графическая оболочка сочетает в себе различные инструменты и функции, которые реализуют следующие возможности:

- навигация по иерархическим и гипертекстовым ссылкам;

- поиск и просмотр информационных материалов, статей и отчетов;

- просмотр графической и видеоинформации, включая поверхности и сетки;

- $\quad$ просмотр баз данных и справочных подсистем;

- $\quad$ выбор вычислительного модуля и создание проекта для работы с ним;

- $\quad$ подготовка к запуску вычислительного модуля (предварительный просмотр и редактирование начальных данных, выбор и предварительный просмотр сетки и т.д.);

- $\quad$ управление расчетом: запуск, просмотр текущих результатов, остановка вычислений;

- просмотр текстовых и графических результатов расчетов;

- $\quad$ запуск внешних приложений (редакторы таблиц, графические пакеты и т.д.);

- $\quad$ настройка среды управления расчетом;

- $\quad$ аутентификация пользователя и контроль доступа к данным;

- $\quad$ накопление новых результатов в специальной базе данных;

- $\quad$ средства для выполнения вычислений на удаленной вычислительной системе.

\section{3. Архитектура интерактивной информационно-расчетной системы}

Архитектура ИИРС основана на идее разделения графической оболочки и расчетно-информационного наполнения. Графическая оболочка включает в себя перечисленные выше инструменты и функции и отделена от содержимого ИИРС. Расчетно-информационное наполнение комплекса задается с помощью специальных конфигурационных файлов в формате XML, организованных иерархическим образом. Эти файлы описывают структуру ИИРС, внешний вид и расположение графических элементов на экране, значки и их основные функции, меню и другие настройки графического интерфейса. Механизм и детали запуска прикладных программ, такие, как начальные данные, номенклатура и типы входных и выходных 
файлов, сетки, вычислительные модули и сценарии для их запуска, описываются в дополнительных конфигурационных файлах.

Таким образом, набор конфигурационных файлов вместе с каталогами, содержащими все информационные и предметные файлы, полностью описывают структуру Системы и все сценарии работы. Изменения и дополнения в системе обеспечиваются редактированием либо реконфигурацией настроечных файлов и не требуют модификации программного кода диалоговой системы. Благодаря этому легко обеспечивается расширение и развитие системы: добавление новых прототипов и информационных материалов, усложнение сценариев работы, поддержка вызова внешних приложений и т.д. Кроме того, отделение графической оболочки от данных позволяет реализовать несколько вариантов ИИРС с различным назначением и информационным содержанием в соответствии с требованиями отдельных пользователей.

\section{4. Описание графического интерфейса и режимов работы}

Графическая оболочка ИИРС поддерживает два основных режима работы - информационный и расчетный. После инициализации и аутентификации пользователя система работает в информационном режиме.

\section{1. Информационный режим}

В этом режиме экран разбивается на два поля - верхнее (поле меню), содержащее заголовок экрана и значки (иконки) с указанием выбираемых объектов (например, прототипов летательных аппаратов), и нижнее (информационное поле), предназначенное для описания этих объектов и действий над ними, а также для вывода информационных материалов (рис. 1 и 2). Нажатие на иконку или кнопку позволяет загрузить другой экран (более низкого уровня) с аналогичной структурой либо выполнить определенное действие (предварительный просмотр сетки или поверхности, статьи или какого-то другого информационного материала, изображения или видео, открытие проекта для выполнения вычислений и т.д.). Также доступны несколько кнопок навигации (возврат в предыдущее окно илив начальный экран и т.п.).

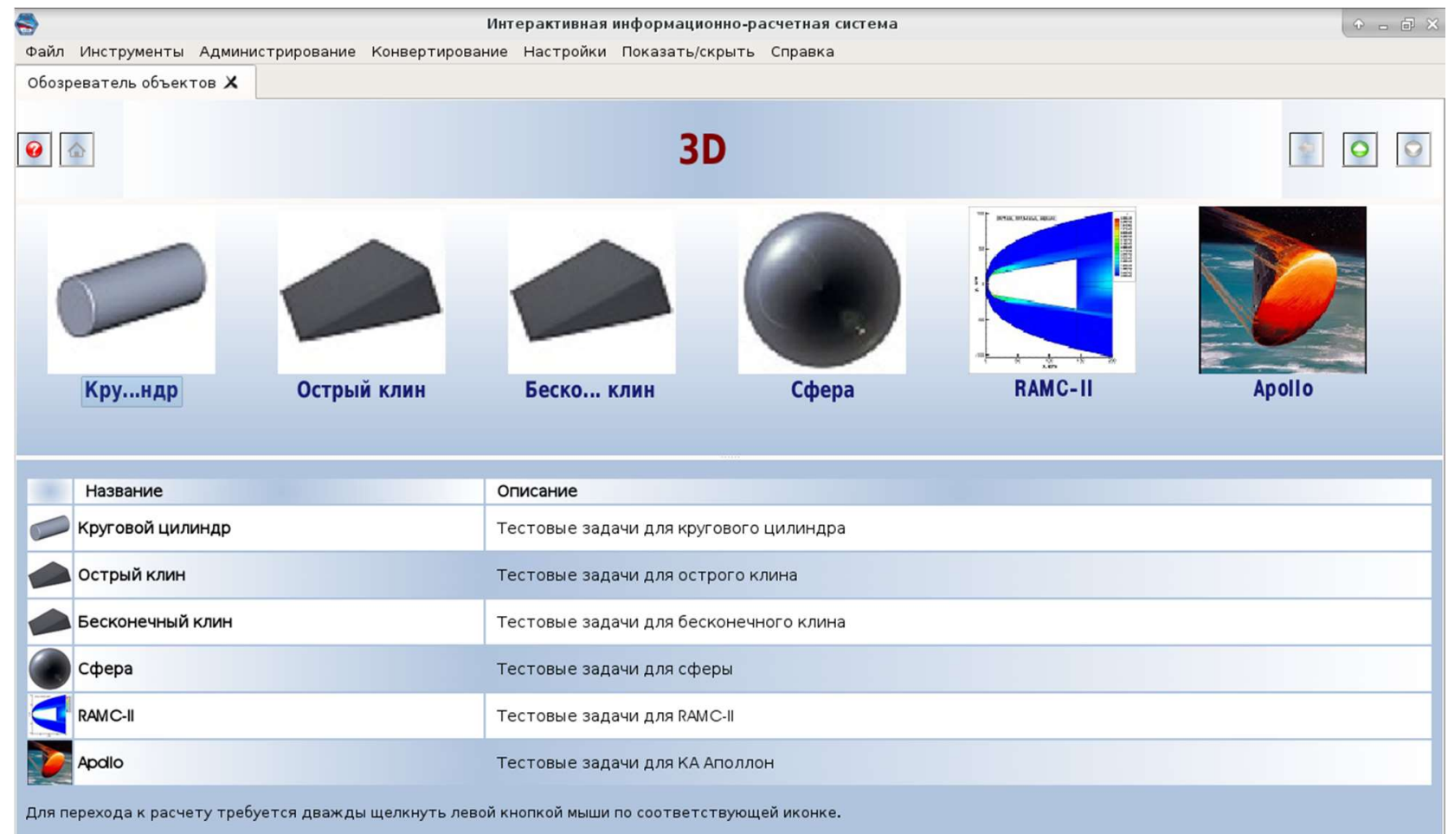

Рис. 1. Пример информационного режима. Нажатие на иконку в поле меню (например, «Острый клин») позволяет перейти к выбору расчетного модуля и запуску его на счет 


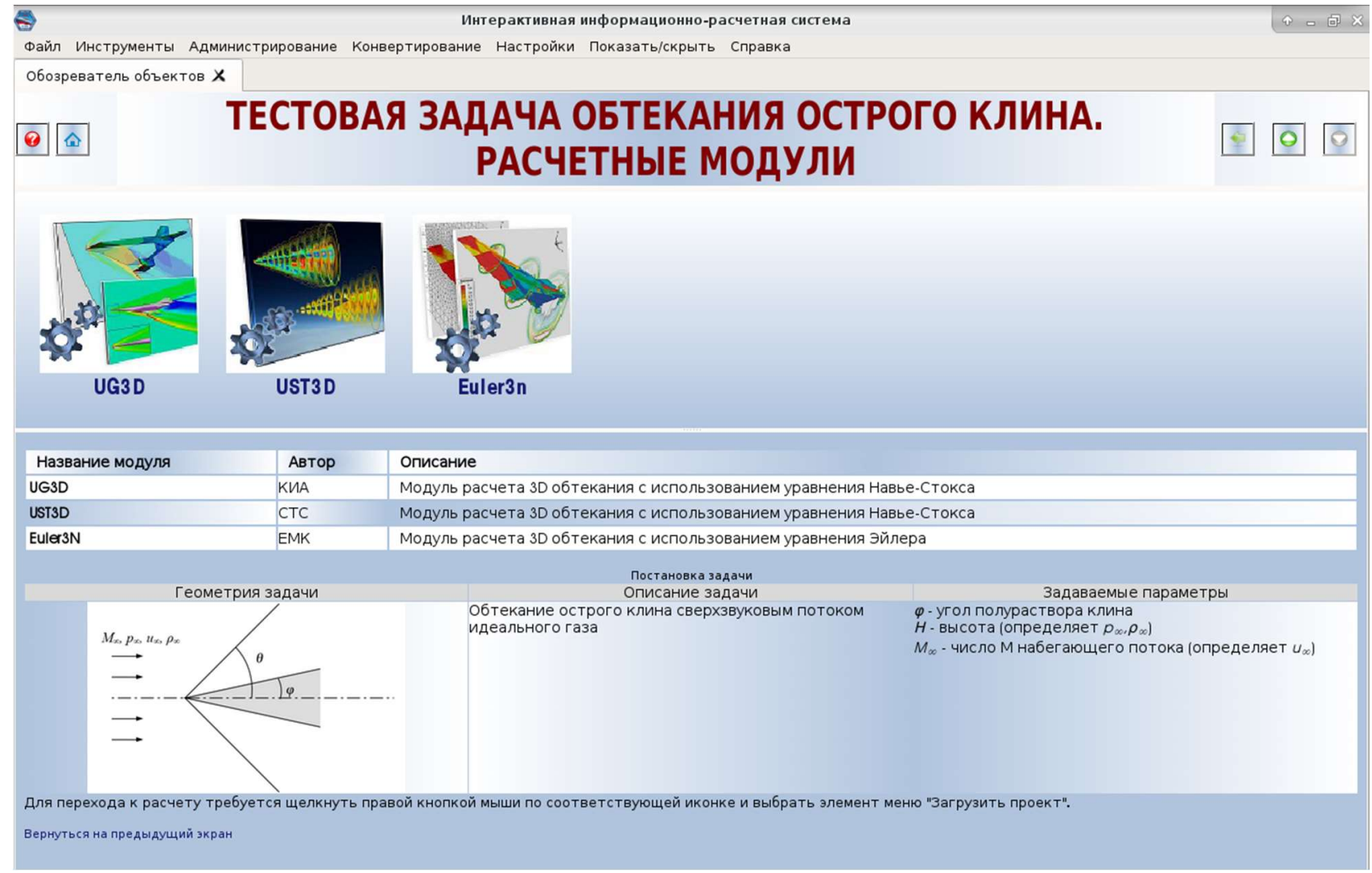

Рис. 2. Пример информационного режима. Экран с расчетными модулями для тестовой задачи сверхзвукового обтекания острого клина. Нажатие на иконку в поле меню (например, «UG3D») загружает экран с тестовыми результатами, щелчок правой кнопкой мыши переключает в расчетный режим.

Представленные элементы управления обеспечивают навигацию в иерархическом информационном пространстве ИИРС. Благодаря этим элементам пользователь может просматривать информацию и иллюстративные материалы по аппарату, предыдущие результаты расчетов или экспериментов, а также перейти в расчетный режим.

Структура и содержание информационного пространства отображаются в аналогичной иерархической структуре файлов конфигурации. Каждый каталог в иерархии содержит файл XML «dirinfo.dif» с полным описанием каждого элемента или поля на экране (его расположение, значок, заголовок, вызываемое действие, имя файла с содержимым информационного поля, ссылка на каталоги нижнего уровня, гипертекстовые ссылки на другие элементы и т.д.).

\section{2. Расчетный режим}

После нажатия на соответствующий значок на информационном экранесо списком вычислительных модулей система переходит в расчетный режим (рис. 3). В этом режиме в левой части экрана появляется новое поле - дерево описания проекта. Оставшаяся часть экрана сохраняет прежнюю структуру с информационным полем и полем меню.

Дерево описания проекта содержит элементы двух типов - информационные поля (пассивные) и элементы действия (активные). Элементы могут быть организованы в иерархическую структуру с возможностью скрывать поддеревья (аналогично структуре дерева файлов в популярных файловых менеджерах). Нажатие на активный элемент (двойной щелчок мыши) вызывает определенную функцию, например, инициализацию и создание рабочего каталога, выбор начальных параметров, выбор и просмотр расчетной сетки и, наконец, запуск расчета (вычислительного модуля) либо его этапа (препроцессора, постпроцессора).

По завершении счета в дереве описания проекта появляются дополнительные элементы, соответствующие файлам результатов и предназначенные для их визуализации (рис. 4). 


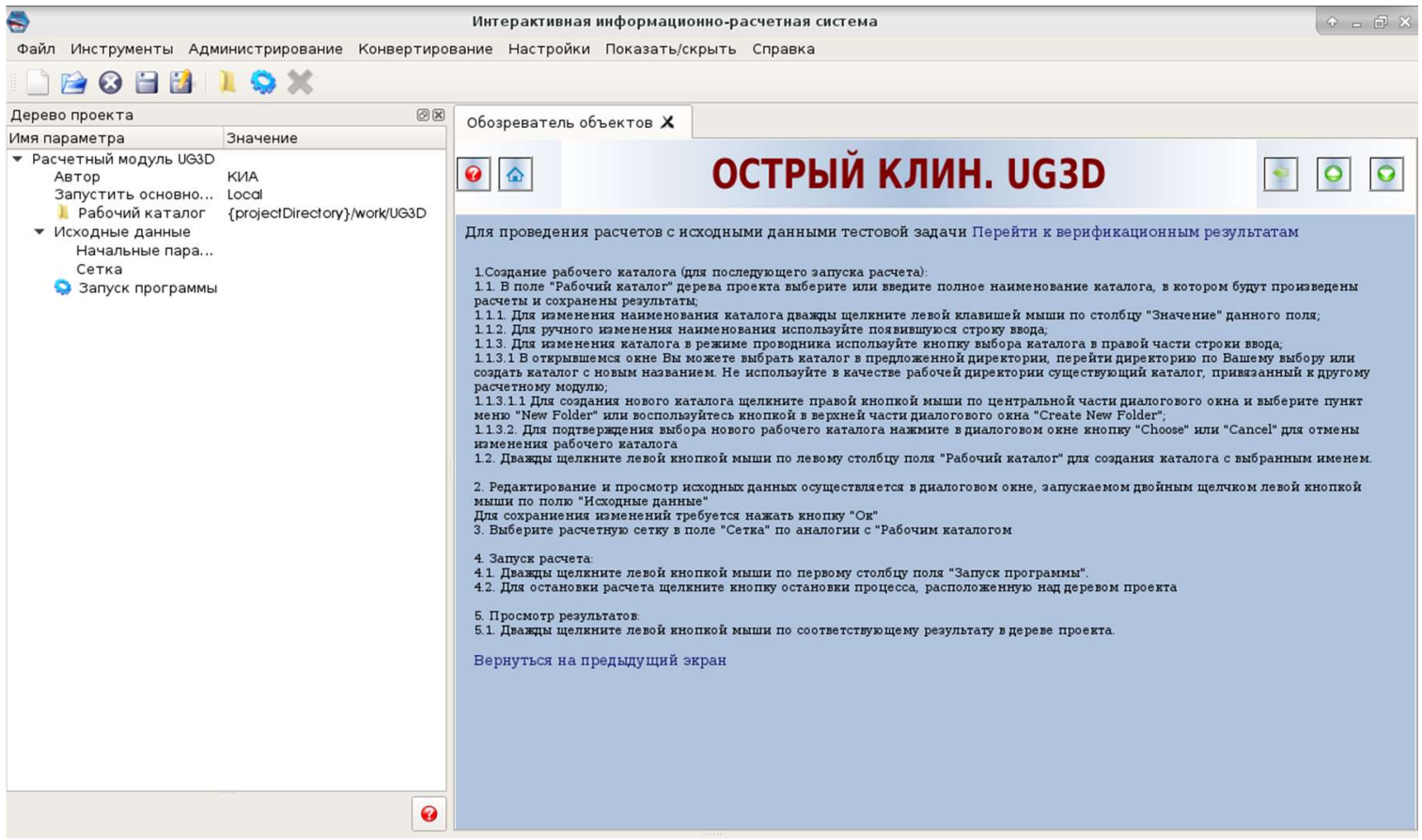

Рис. 3. Пример расчетного режима. Выбранный расчетный компьютерный код реализует модель вязкого сжимаемого теплопроводного газа, описываемую пространственной нестационарной системой уравнений Навье - Стокса. Элементы в дереве описания проекта (слева) представляют собой параметры расчета: имя автора, рабочий каталог, входные файлы или диалоговые окна для задания начальных данных, файл сетки и кнопку действия для запуска вычисления. Текст в информационном поле (справа) описывает процедуру проведения расчета, а также может содержать элементы для выбора сетки

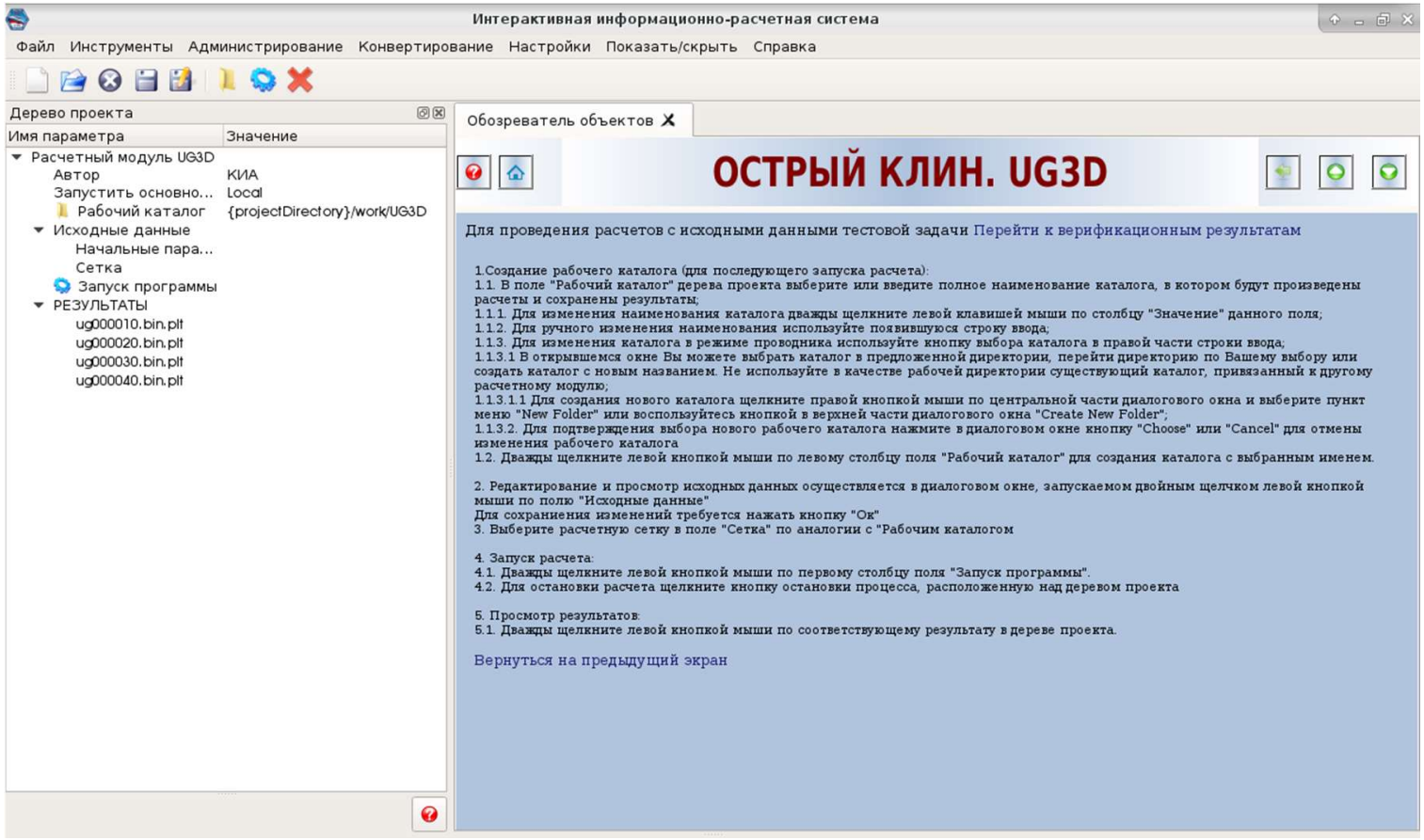

Рис. 4. Пример расчетного режима.После завершения расчета в дереве описания проекта (слева) под заголовком «РЕЗУЛЬТАТЫ» появились элементы, соответствующие файлам результатов. В данном примере эти элементы имеют расширение имени файла «plt», что соответствует формату входных данных для графической программы ParaView

Нажатие на такой элемент запускает программу графического вывода - встроенный в ИИРС графический визуализатор либо внешнюю графическую программу (ParaView, 
Gnuplot, TecPlot и т.п., в соответствии с типом элементаи настройками) для вывода результаты вычислений (рис. 5). После просмотра этих результатов пользователь может повторить подготовительные действия (например, изменить входные данные) и снова запустить расчет.

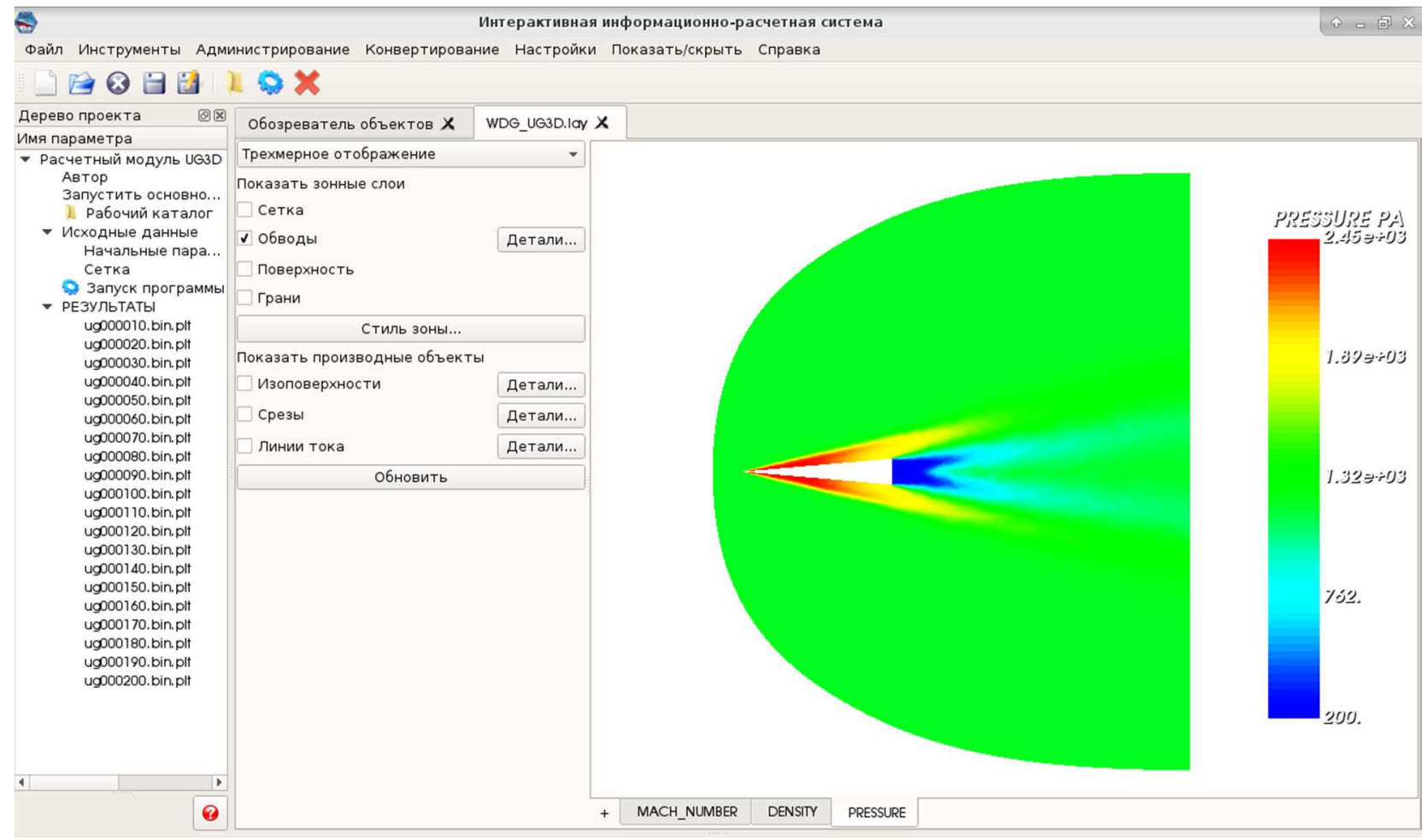

Рис. 5. Пример визуализации результатов расчета для объекта «Острый клин», полученный после нажатия на соответствующий элемент в дереве описания проекта. Рисунок получен с помощью встроенного в ИИРС графического визуализатора

Элементы проекта описываются специальными конфигурационными файлами в формате XMLc расширениями «prj» и «prs». Эти файлы содержат всю необходимую информацию о каждом элементе, такую, как подписи, вызываемые действия и ссылки на внешние файлы.

Механизм, основанный на дереве описания проекта, позволяет обеспечить богатую функциональность и гибкость при организации сценариев работы. Кроме того, этот механизм позволяет вынести ряд функций из графической оболочки в командные файлы (скрипты), а также организовать проведение расчетов во временных рабочих каталогах, что важно для поддержки многопользовательских и многозадачных режимов работы.

Приоткрытом дереве описания проекта сохраняется возможность работы с информационным полем (в правой части экрана). При нажатии на соответствующую кнопку навигации система может закрыть дерево описания проекта и вернуться в информационный режим.

\section{3. Другие режимы работы системы}

ИИРС также реализует режимы работы со следующими подсистемами:

- $\quad$ специализированных баз данных под управлением СУБД Postgre SQL, содержащие данные кинетики горения, кинетических параметров и коэффициентов переноса, спектрально-оптических характеристик, физико-химической кинетики, химических реакций, химической кинетики высокотемпературных газов и некоторых других;

- $\quad$ интерактивной помощи, содержащей описание элементов ИИРС и механизмов работы с ними;

- утилит для конвертирования сеток между различными форматами и их интерактивнойвизуализации; 
- $\quad$ средств администрирования и контроля доступа для управления списком пользователей и разграничения уровня доступа к данным ИИРС;

- $\quad$ средств для настройки параметров системы и предпочтений пользователей (например, для выбора утилит для работы с различными типами файлов), а также для редактирования конфигурационных файлов.

\section{3. Сценарий работы в системе}

На рис. 6 приведена диаграмма, иллюстрирующая структуру ИИРС, а также пример сценария работы в системе. В этом примере пользователь выбирает спускаемый космический аппарат Space Shuttle из набора прототипов Р1, указывает малую сетку из имеющегося набора для этого аппарата, а затем выбирает расчетный код 1 и переходит в расчетный режим. Элементы дерева проекта в этом режиме иллюстрируют дальнейшие шаги для запуска расчета и просмотра результатов.

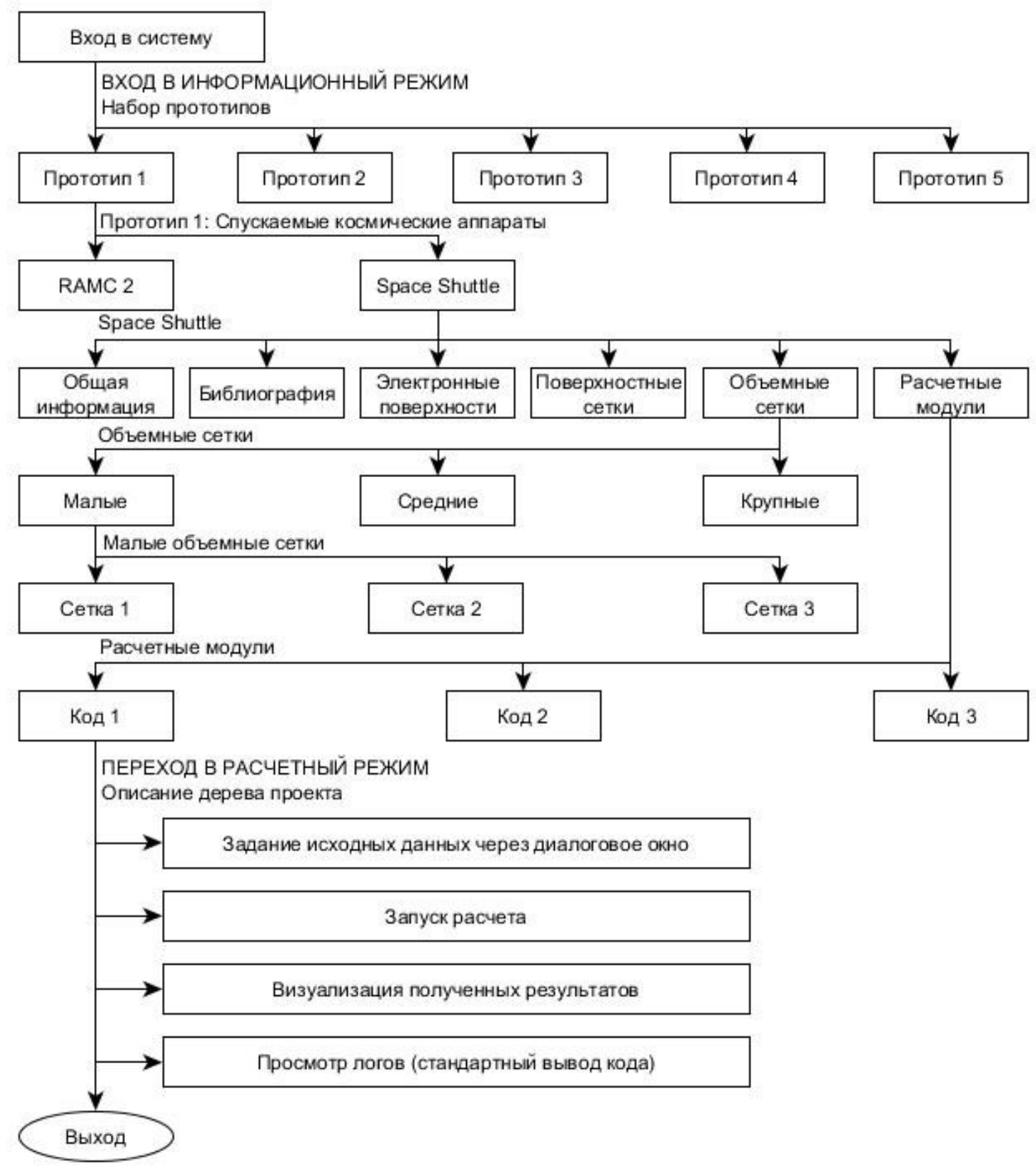

Рис. 6. Пример сценария работы в ИИРС 


\section{5. Реализация интерактивной графической оболочки}

Графическая оболочка ИИРС написана на языке C ++ с использованием кроссплатформенной прикладной среды с открытым исходным кодом Qt $[13,14]$ версии 5.5.1.

Программа может работать как на операционной системе Microsoft Windows, так и в среде Linux. Использование конструкции "ifdef" и "ifndef" позволяет реализовать системнозависимые части кода для каждой операционной системы, что обеспечивает унификацию исходного кода. Общая длина текущего варианта программы превышает 5000 строк исходного кода.

Вычислительные модули могут быть написаны на любых языках программирования и скомпилированы любыми подходящими компиляторами. Модули запускаются через командные файлы (скрипты). Подготовлены два набора скриптов - для OC Windows (файлы с расширением «bat») и для OC Linux (скрипты оболочки bash). Если для какого-либо модуля требуется подключение специализированной библиотеки, необходимая среда запуска может быть прописана в соответствующем скрипте. Соответственно, сложная процедура запуска может быть также реализована посредством скрипта (например, параллельный расчет с использованием среды Оpen MP и / или MPI). Подход, основанный на использовании командных файлов, позволяет, как правило, освободить оболочку от выполнения сложных действий и специфических настроек.

\section{6. Заключение}

Представленная интерактивная информационно-расчетная система и ее основной элемент, графическая оболочка, обеспечивают интеграцию вычислительных модулей, инструментов для работы с расчетными сетками, механизмов проведения расчетов, информационных ресурсов и других элементов в единую информационно-вычислительную среду. Дальнейшее развитие системы осуществляется путем расширения возможностей графической оболочки и добавления новых вычислительных модулей вместе с соответствующими информационными элементами (руководства по эксплуатации, статьи, результаты вычислений). ИИРС станет эффективной и удобной технологической и образовательной средой для выполнения вычислений, визуализации и анализа результатов при решении задач газовой динамики и проведении междисциплинарных исследований.

\section{Благодарности и ссылки на гранты}

Работа поддержана средствами государственного бюджета по госзаданию АААА-А17117021310372-6. Авторы благодарят к.ф.-м.н. Х.Х. Ильясова за помощь в работе и д.ф.-м.н., профессора, академика РАН С.Т. Суржикова за руководство работой, разработку концепции и основополагающего предметно-ориентированного сценария ее работы.

Работа выполнена по теме государственного задания (№ госрегистрации АAАA-A20120011690135-5) и при поддержке гранта РФФИ № 19-01-00515.

\section{Литература}

1. Суржиков С.Т. Перспективы многоуровнего подхода к задачам компьютерной аэрофизики// /Физико-химическая кинетика в газовой динамике. 2008. T. 7. http://chemphys.edu.ru/issues/2008-7/articles/435

2. Суржиков С.Т. Радиационный нагрев поверхности сверхорбитальных спускаемых космических аппаратов с учетом атомных линий//Физико-химическая кинетика в газовой динамике. 2014. T. 15. http://chemphys.edu.ru/issues/2014-15-4/articles/235

3. Candler G.V., MacCormack R.W. Computation of weakly ionized hypersonic flows in thermochemical nonequilibrium //J. Thermophysics. 1991. Vol. 5. Pp. 266-273. 
4. Grasso F., Capano G. Modeling of ionizing hypersonic flows in nonequilibrium //J. Spacecraft Rockets. 1995. Vol. 32. Pp. 217-224.

5. Josyula E., Bailey W.F. Governing equations for weakly ionized plasma flowfields of aerospace vehicles //J. Spacecraft Rockets. 2003. Vol. 40. Pp. 845-857.

6. Железнякова А.Л., Суржиков С.T. Поле течения около космического аппарата FIRE II под углом атаки//Физико-химическая кинетика в газовой динамике. 2010. T.9. http://chemphys.edu.ru/issues/2010-9/articles/139

7. Surzhikov S.T. Radiative-Convective Heat Transfer of a Spherically Shaped Space Vehicle in Carbon Dioxide //High Temperature. 2011. Vol. 49. No. 1. Pp. 92-107.

8. Surzhikov S.T. Coupled radiative gasdynamic interaction and non-equilibrium dissociation for largescale returned space vehicles //Chemical Physics. 2012. Vol. 398. Pp. 56-63.

9. Shang J.S., Surzhikov S.T. Nonequilibrium radiative hypersonic flow simulation //Progress in Aerospace Sciences. 2012. Vol. 53. Pp. 46-65.

10. Surzhikov S.T. Radiative-collisional models in non-equilibrium aerothermodynamics of entry probes //Journal of Heat Transfer. 2012. Vol. 134.031002.

11. Surzhikov S.T. Two-dimensional numerical analysis of flow ionization in the RAM-C-II flight experiment //Russian J. Physical Chemistry B. 2015.Vol. 9. Pp. 69-86.

12. Ермаков М.К., Железнякова А.Л., Котов М.А. и др. Разработка информационно-компьютерной системы моделирования аэрофизики высокоскоростных аппаратов КПВМ АВАРС // Материалы XXIX Научно-техническая конференции по аэродинамике. - Жуковский: Жуковский. 2018. - C. 124.

13. https://www.qt.io/

14. Dalheimer M.K. Programming with Qt: Writing Portable GUI Applications on Unix and Win32. O'Reilly Media. 2002.

\section{References}

1. Surzhikov S.T. Prospects of the multi-scale approach to the computer agrophysics problems//PhysicalChemical Kinetics in Gas Dynamics. 2008. V. 7. http://chemphys.edu.ru/issues/2008-7/articles/435

2. Surzhikov S.T. Radiative heating of the super-orbital re-entry space vehicles in view of atomic lines // Physical-ChemicalKineticsinGasDynamics. 2014. V. 15. http://chemphys.edu.ru/issues/2014-15-4/articles/235

3. Candler G.V., MacCormack R.W. Computation of weakly ionized hypersonic flows in thermochemical nonequilibrium //J. Thermophysics. 1991. Vol. 5. Pp. 266-273.

4. Grasso F., Capano G. Modeling of ionizing hypersonic flows in nonequilibrium //J. Spacecraft Rockets. 1995. Vol. 32. Pp. 217-224.

5. Josyula E., Bailey W.F. Governing equations for weakly ionized plasma flowfields of aerospace vehicles //J. Spacecraft Rockets. 2003. Vol. 40. Pp. 845-857.

6. Zheleznyakova A.L., Surzhikov S.T. Flowfield around FIRE II space vehicle under angle of attack // Physical-Chemical Kinetics in Gas Dynamics. 2010. V. 9. http://chemphys.edu.ru/issues/2010-9/articles/139

7. Surzhikov S.T. Radiative-Convective Heat Transfer of a Spherically Shaped Space Vehicle in Carbon Dioxide //High Temperature. 2011. Vol. 49. No. 1. Pp. 92-107.

8. Surzhikov S.T. Coupled radiative gasdynamic interaction and non-equilibrium dissociation for largescale returned space vehicles //Chemical Physics. 2012. Vol. 398. pp. 56-63.

9. Shang J.S., Surzhikov S.T. Nonequilibrium radiative hypersonic flow simulation //Progress in Aerospace Sciences. 2012. Vol. 53. Pp. 46-65.

10. Surzhikov S.T. Radiative-collisional models in non-equilibrium aerothermodynamics of entry probes //Journal of Heat Transfer. 2012. Vol. 134.031002. 
11. Surzhikov S.T. Two-dimensional numerical analysis of flow ionization in the RAM-C-II flight experiment //Russian J. Physical Chemistry B. 2015.Vol. 9. Pp. 69-86.

12. Ermakov M.K., Zheleznyakova A.L., Kotov M.A. et. all. Development of information and computer system CPVM AHARS for modeling of high-speed vehicles aerophysics// Materials ofXXIXScientific and technical conference on aerodynamics. — Zhukovsky: Zhukovsky. 2018. P.124.

13. https://www.qt.io/

14. Dalheimer M.K. Programming with Qt: Writing Portable GUI Applications on Unix and Win32. O'Reilly Media. 2002.

Статья поступила в редакцию 4 марта 2020 г. 